Article

\title{
Real-Time Control Strategy for Aggregated Electric Vehicles to Smooth the Fluctuation of Wind-Power Output
}

\author{
Zicong Yu ${ }^{1,2}$, Ping Gong ${ }^{1,2}$, Zhi Wang ${ }^{1,2}$, Yongqiang Zhu ${ }^{2, *}$, Ruihua Xia ${ }^{2}$ and Yuan Tian ${ }^{2}$ \\ 1 State Key Laboratory of Operation and Control of Renewable Energy \& Storage System, China Electric \\ Power Research Institute Co., Ltd., Beijing 100192, China; yuzicong@ncepu.edu.cn (Z.Y.); \\ m17710605275@163.com (P.G.); a13976439377@163.com (Z.W.) \\ 2 State Key Laboratory of Alternate Electrical Power System with Renewable Energy Sources, North China \\ Electric Power University, Beijing 102206, China; supplyports@sina.com (R.X.); \\ Yuan.Tian966@outlook.com (Y.T.) \\ * Correspondence: zyq@ncepu.edu.cn; Tel.: +86-139-1000-2860
}

Received: 31 December 2019; Accepted: 8 February 2020; Published: 9 February 2020

\begin{abstract}
Electric vehicles (EVs) are flexible demand-side response resources in a power distribution system. Reasonable and orderly control of charging/discharging processes of aggregated EVs can improve their coordination and interaction with the distribution system and ensure its efficient and stable operation. Aiming at the problem that the fluctuation of wind power output may affect the stable operation of distribution system, a real-time control strategy for aggregated EVs to smooth the fluctuation of wind power is proposed. Firstly, considering the dispatchability of EVs, the charging/discharging energy boundary model is established to determine the charging/discharging margin of an EV at each moment. Then, first-order low-pass filtering is used to determine the total dispatching power of aggregated EVs. Finally, the total charging power of aggregated EVs is determined and power allocation is carried out. Simulation results show that the proposed strategy can achieve real-time smoothing for the fluctuation of wind power output while meeting the charging requirements of EVs, and the proposed strategy can not only reduce the fluctuation rate of total load, but also realize peak shaving and valley filling for the distribution system.
\end{abstract}

Keywords: aggregated electric vehicles; real-time control; wind power; charging/discharging energy boundary model; first-order low-pass filtering

\section{Introduction}

Renewable energy sources (RES) represented by wind power generation and photovoltaic power generation are an important way to alleviate the increasingly prominent environmental pollution, traditional energy shortages and other problems [1] and have been developed vigorously in recent years. However, due to the obvious intermittency, volatility and uncertainty of RES, with the increasing proportion of its connection to the power grid, the safe and stable operation of the power grid may be adversely affected. With the continuous increasing of the market share of electric vehicles (EVs), their role as a flexible demand-side response resource in the operation and regulation of distribution system has become increasingly prominent. The complementary use of EVs with RES contributes to the economic and environmental benefits of its overall operation: (1) RES can reduce the dependence on fossil fuels and accomplish low-carbon in the true sense [2]; (2) EVs can also help solve the intermittent problem of RES and reduce the cost of the energy-storage system [3]; (3) the local consumption and utilization of RES by EVs can be realized through the micro power grid, and the side effects caused by their separate connection to the power grid can be reduced. 
In recent years, several studies have been carried out on the joint optimal dispatching of the integration of EVs and RES in the power systems. On the basis of traditional day-ahead power generation plan, Sun et al [4] proposed an optimal scheduling method of aggregated EVs on a multi-time scale, improving the utilization rate of wind power and enhancing the clean charge of EVs. Cheng et al [5] proposed an equivalent quantity model of EV charging on the basis of the probabilistic model, and realized the cooperative operation of EVs and wind power. Zhao et al [6] proposed an economic dispatch model of aggregated EVs and wind power solving by an optimization algorithm based on the well-established particle swarm optimization (PSO) and interior point method. Vasirani et al [7] made wind power generators fully participate in electricity markets by forming a virtual power plant (VPP) with EVs that can store the energy to overcome the intermittent nature of the energy supply. For integrated energy systems that include a large number of EVs, Li et al [8] proposed a coordinated scheduling model that can effectively improve the consumption rate of renewable energy and reduce the volatility of renewable energy by considering the coordination of electric vehicles, tie lines, and heating/cooling systems in multiple functional areas. Zhang et al [9] proposed a novel unit commitment model considering demand response and EVs, and solved it through the fuzzy opportunity limitation program. The numerical study shows that the model can promote the utilization of wind power evidently, making the power system operation eco-friendlier and more economical.

At present, most of the research on the participation of aggregated EVs in the power grid scheduling belong to the optimal scheduling in large timescales, which depends on accurate prediction of uncertain factors [10]. However, there are many uncertainties in the power system that are difficult to predict accurately in advance, such as renewable energy output and EV charge loads. There is a low prediction accuracy of renewable energy output in large timescales. For example, the day-ahead prediction of wind power and photovoltaics can only predict the overall output trend the next day, and the real-time output usually has a fluctuation component. The charge process of EVs is random and uncertain, and the reported charge plan for the next day may be changed due to some emergencies. Therefore, it is necessary to control the aggregated EVs in real time to avoid prediction error.

There are some research works on the real-time control of aggregated EVs now: Deilami et al [11] proposed a real-time smart load management (RT-SLM) control strategy for the charging process of aggregated EVs in a smart grid, realizing the minimization of the total cost of generating the energy plus the associated grid energy losses. Luo et al [12] designed a simple but effective EV-charging scheduling scheme for real-time coordination of randomly arriving or departing EVs in low-voltage residential networks. Zhang et al [13] proposed a charging and discharging dispatch strategy for EVs on the basis of fuzzy control, but its control accuracy is not high. Aiming at residential aggregated EVs, Su et al [14] and Wang et al [15] used time-of-use electricity prices as the background to enable EVs to discharge during peak-load periods and charge during valley-load periods to achieve peak shaving and valley filling, but this will cause sudden load change at the beginning and end of the valley period. Hu et al [16] proposed a real-time control strategy for aggregated EVs to smooth out photovoltaic fluctuations, but this real-time control strategy is actually a real-time correction of the day-ahead scheduling error. Pahasa et al [17] used multiple model predictive control (MMPC) to manage the charging process of EVs in real time, but this relied on short-term load prediction. In conclusion, there are few real-time control strategies for aggregated EVs that can adapt to scenarios where uncertain factors are difficult to predict. Some studies rely on prediction technology to achieve real-time rolling optimization.

Given the above background, a real-time control strategy for aggregated EVs to smooth fluctuation of wind power output is proposed in this paper. Firstly, in order to make full use of the scheduling capability of EV, the charging/discharging energy boundary model is established, which can be used to calculate the adjustable range of charging and discharging power of EVs at the current moment. Then, a method to determine the total charging power of aggregated EVs is proposed, which is based on the first-order filtering algorithm and can achieve the goal of smoothing the total load curve of distribution system. Finally, the power allocation method of aggregated EVs is proposed, which optimizes the 
charging power of each EV by maximizing the total charging/discharging margin of the aggregated EVs at the following moment, and converts the problem into a mixed-integer linear programming (MILP) problem by using the piecewise linearization method, which improves the solution efficiency.

\section{The Control Framework of Aggregated Electric Vehicles (EVs) Participating in Power Grid Scheduling}

The output power of a wind farm fluctuates due to the uncertainty and intermittence of wind, and the wakes between wind turbine units. Large-scale wind power integration may cause load fluctuation and affect the stable operation of the power system. As a flexible decentralized dispatchable unit, EVs can coordinate and complement wind power under certain conditions. The coordinated management of aggregated EVs with effective control strategies can smooth the load curve, promote wind-power accommodation, and ensure the safe and reliable operation of the power system [18].

The real-time control framework of aggregated EVs participating in power grid scheduling is shown in Figure 1. All charging piles in the figure are direct current (DC) charging piles whose charging and discharging power can be adjusted continuously between 0 and the maximum [19,20]. At the same time, this kind of charging pile has the function of reading information, such as the time when the EV is connected to the charging pile (on-grid time), the time when the EV leaves the charging pile (off-grid time), and the battery level of the EV, etc. It can also perform information exchange with the aggregator management center (AMC) through the low-voltage carrier communication module. The AMC can model the charging process of the aggregator and send the state information of the aggregator to the load-management control center (LMCC). LMCC collects the status information of each AMC in real time, and calculates the charging power target of every aggregator by a real-time control strategy. When AMC receives instructions from the upper controller, it can respond in real time by orderly controlling the charging power of each EVs in the power grid.

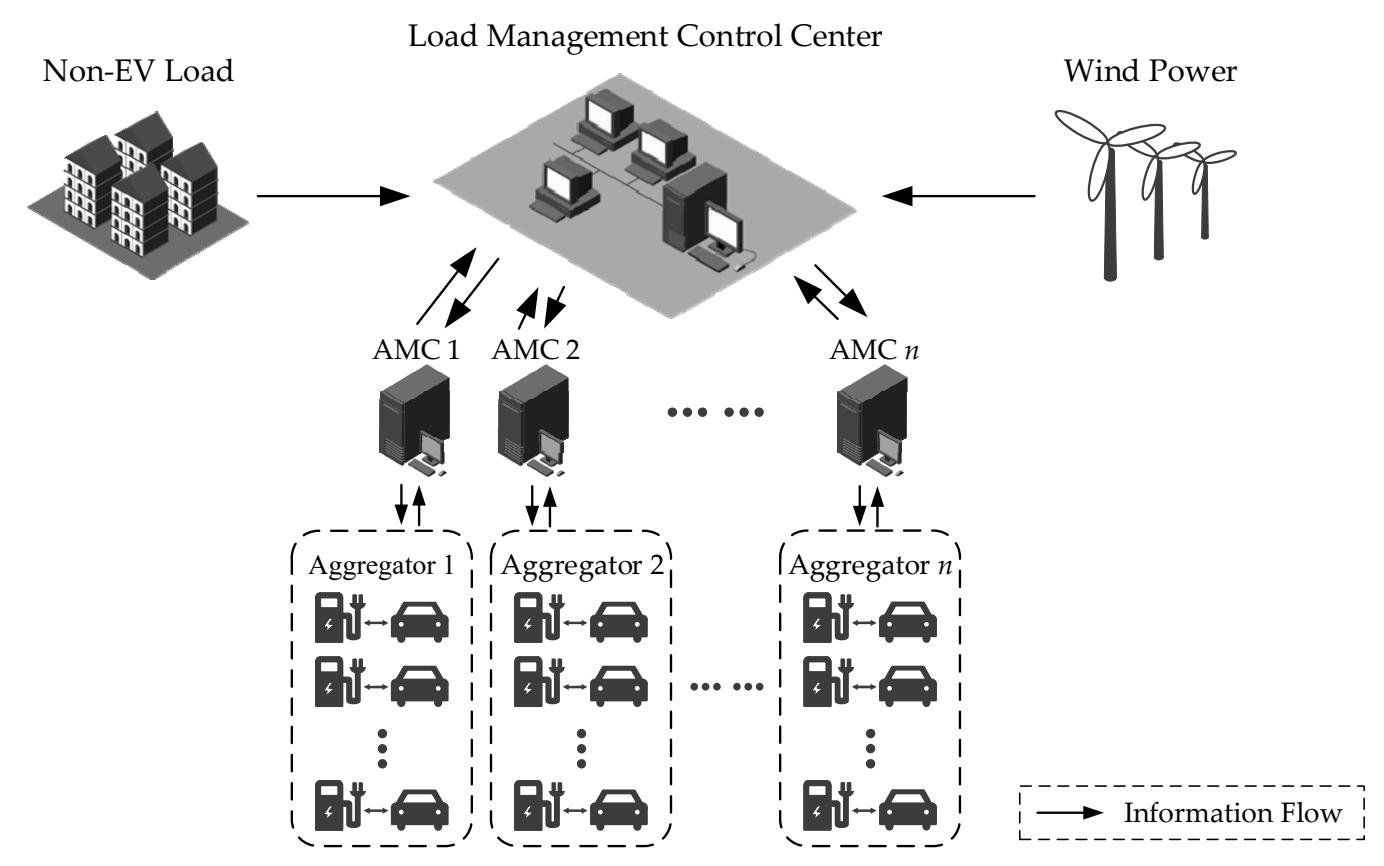

Figure 1. Schematic illustration of the real-time control framework.

According to the willingness of EV users to participate in grid scheduling, EVs can be divided into three categories:

(1) Do not participate in grid scheduling. An EV not subject to the schedule in the charging process. When the EV is connected to the charging pile, it is immediately charged at the rated maximum charging power until the EV leaves the charging pile or the EV battery reaches the set target value. 
(2) Participate in grid scheduling but not allowed to discharge to grid. This process can be called grid-to-vehicle (G2V).

(3) Participate in grid scheduling and allow discharge to the vehicle. This process can be called vehicle-to-grid (V2G).

\section{The Charging/Discharging Energy Boundary Model of EVs}

In order to effectively control the charging/discharging processes of EVs participating in power grid scheduling, this paper first establishes a charging/discharging energy boundary model [21].

Assume that the maximum charging and discharging power of EV $i$ are $P_{\mathrm{cmax}}^{i}$ and $P_{\mathrm{dmax}}^{i}, \eta_{\mathrm{c}}^{i}$ and $\eta_{\mathrm{d}}^{i}$ respectively represent charging and discharging efficiency. When the EV $i$ is connected to the grid at time $t_{\text {in }}^{i}$, the state of charge (SOC) of the EV battery is $E_{\text {in }}^{i}$. At the same time, the EV user sets the off-grid time $t_{\text {out }}^{i}$ and the SOC they expect called $E_{\text {out }}^{i}$. Then, the connected duration $T_{\text {set }}^{i}$ is defined as:

$$
T_{\mathrm{set}}^{i}=t_{\mathrm{out}}^{i}-t_{\text {in }}^{i}
$$

Define the standard charging duration $T_{\mathrm{Nc}}^{i}$ of $\mathrm{EV} i$ as:

$$
T_{\mathrm{Nc}}^{i}=\frac{\left(E_{\max }^{i}-E_{\mathrm{in}}^{i}\right) B^{i}}{\eta_{\mathrm{c}}^{i} P_{\mathrm{cmax}}^{i}}
$$

where $B^{i}$ is the battery capacity and $E_{\max }^{i}$ is the maximum SOC of EV $i$.

If $T_{\text {set }}^{i} \leq T_{\mathrm{Nc}^{\prime}}^{i}$ the EV $i$ is uncontrollable, and it will immediately be charged at the maximum charging power until it leaves the grid. If $T_{\mathrm{set}}^{i}>T_{\mathrm{Nc}^{\prime}}^{i}$ the $\mathrm{EV} i$ is controllable, and its charging power can be adjusted continuously.

For a controllable EV, the charging/discharging energy boundary model is shown in Figures 2 and 3 , where $E_{\min }^{i}$ is the minimum SOC of the EV $i$.

For a controllable EV in G2V: the EV will be charged at its maximum charging power immediately from the moment it is connected to the grid, and the battery level remains constant after reaching the maximum SOC so that we can get the trajectory of $\mathrm{SOC}(\mathrm{A} \rightarrow \mathrm{B} \rightarrow \mathrm{C}$ in Figure 1) regarded as the upper boundary of energy; After the EV is connected to the grid, the SOC stays the same, and is then charged at the maximum power to the off-grid moment. The trajectory of this process ( $A \rightarrow I \rightarrow G$ in Figure 1 ) is regarded as the lower boundary of energy.

For a controllable EV in V2G: the upper boundary of energy ( $A \rightarrow B \rightarrow C$ in Figure 2$)$ is the same as a controllable EV in G2V; The EV immediately discharges at the maximum power at the moment it is connected to the power grid, and then it begins to charge at the maximum power until its SOC reaches the user-set target value at off-grid time, the trajectory of this process ( $\mathrm{A} \rightarrow \mathrm{D} \rightarrow \mathrm{F} \rightarrow \mathrm{G}$ in Figure 2$)$ is regarded as the lower boundary of energy.

In Figures 2 and 3, the parameter of the upper boundary of energy $t_{\mathrm{B}}^{i}$ is defined as:

$$
t_{\mathrm{B}}^{i}=t_{\mathrm{in}}^{i}+T_{\mathrm{Nc}}^{i}
$$

In Figure 2, the parameter of the lower boundary of energy $t_{\mathrm{I}}^{i}$ is defined as:

$$
t_{\mathrm{I}}^{i}=t_{\mathrm{out}}^{i}-\frac{\left(E_{\mathrm{out}}^{i}-E_{\mathrm{in}}^{i}\right) B^{i}}{\eta_{\mathrm{c}}^{i} P_{\mathrm{cmax}}^{i}}
$$

In Figure $3 \mathrm{a}$, the parameters of the lower boundary of energy $t_{\mathrm{D}}^{i}$ and $t_{\mathrm{F}}^{i}$ are respectively defined as:

$$
t_{\mathrm{D}}^{i}=t_{\mathrm{in}}^{i}+\frac{\left(E_{\mathrm{in}}^{i}-E_{\text {min }}^{i}\right) B^{i} \eta_{\mathrm{d}}^{i}}{P_{\mathrm{d} \max }^{i}}
$$




$$
t_{\mathrm{F}}^{i}=t_{\mathrm{out}}^{i}-\frac{\left(E_{\mathrm{out}}^{i}-E_{\min }^{i}\right) B^{i}}{\eta_{\mathrm{c}}^{i} P_{\mathrm{cmax}}^{i}}
$$

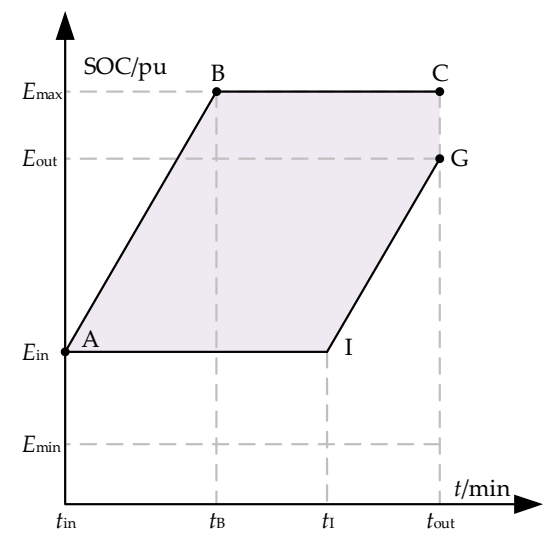

Figure 2. The charging/discharging energy boundary model of a controllable electric vehicle (EV) in grid-to-vehicle (G2V).

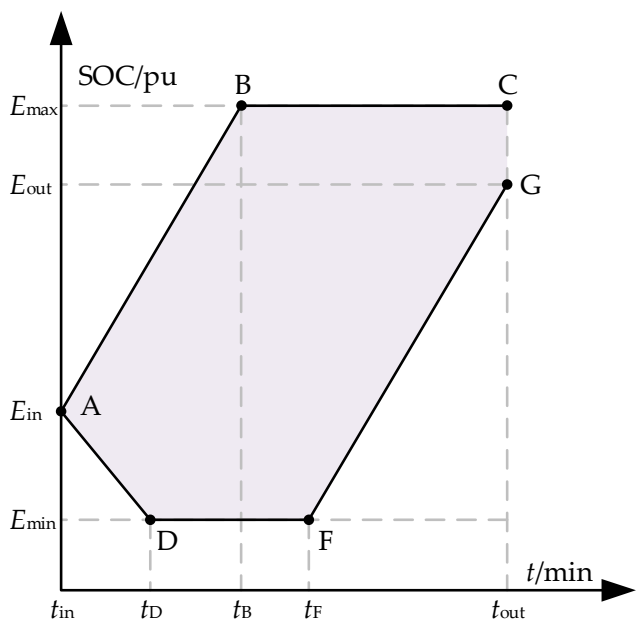

(a)

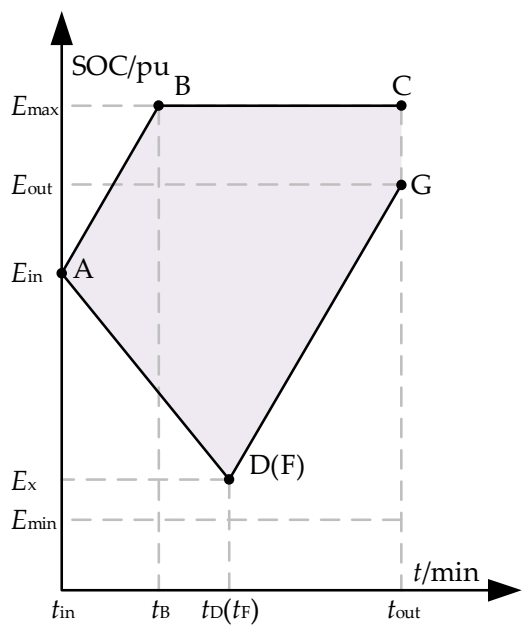

(b)

Figure 3. The charging/discharging energy boundary model of a controllable EV in vehicle-to-grid (V2G): (a) dischargeable to minimum state of charge (SOC, point D does not coincide with point F); (b) undischargeable to the minimum SOC (point $\mathrm{D}$ coincide with point $\mathrm{F}$ ).

In Figure $3 \mathrm{~b}$, the parameters of the lower boundary of energy $t_{\mathrm{D}^{\prime}}^{i} t_{\mathrm{F}}^{i}$ and $E_{\mathrm{x}}^{i}$ are respectively defined as:

$$
\begin{gathered}
t_{\mathrm{D}}^{i}=t_{\mathrm{F}}^{i}=t_{\mathrm{in}}^{i}+\frac{\left(E_{\mathrm{in}}^{i}-E_{\mathrm{x}}^{i}\right) B^{i} \eta_{\mathrm{d}}^{i}}{P_{\mathrm{d} \text { max }}^{i}} \\
E_{\mathrm{x}}^{i}=\frac{E_{\mathrm{in}}^{i} B^{i} \eta_{\mathrm{c}}^{i} \eta_{\mathrm{d}}^{i} P_{\mathrm{cmax}}^{i}+E_{\mathrm{out}}^{i} B^{i} P_{\mathrm{dmax}}^{i}-\left(t_{\mathrm{out}}^{i}-t_{\mathrm{in}}^{i}\right) \eta_{\mathrm{c}}^{i} \eta_{\mathrm{d}}^{i} P_{\mathrm{cmax}}^{i} P_{\mathrm{dmax}}^{i}}{B^{i}\left(\eta_{\mathrm{c}}^{i} \eta_{\mathrm{d}}^{i} P_{\mathrm{cmax}}^{i}+P_{\mathrm{dmax}}^{i}\right)}
\end{gathered}
$$

Set the charging power range of EV $i$ at time $t$ as $\left[-P_{-}^{i}(t), P_{+}^{i}(t)\right]$.

For an uncontrollable EV $i$, it will always be charged at the maximum charging power, that is, $P_{-}^{i}(t)=-P_{\mathrm{cmax}}^{i}, P_{+}^{i}(t)=P_{\mathrm{cmax}}^{i}$.

For a controllable EV, its charging power range will be determined according to its charging/discharging energy boundary model. Take a controllable EV in V2G for example. As shown in Figure $4, E(t)$ is the SOC of EV battery at time $t$. First, determine the upper boundary $E_{+}^{i}(t+\Delta t)$ and 
lower boundary $E_{-}^{i}(t+\Delta t)$ of the SOC at the time $t+\Delta t$ according to the charging/discharging energy boundary model, and calculate the boundary distance of the SOC at the current time:

$$
\begin{aligned}
& e_{+}^{i}(t)=E_{+}^{i}(t+\Delta t)-E^{i}(t) \\
& e_{-}^{i}(t)=E^{i}(t)-E_{-}^{i}(t+\Delta t)
\end{aligned}
$$

where, $e_{+}^{i}(t)$ and $e_{-}^{i}(t)$ are the upper and lower boundary distance of EV $i$ at time $t$.

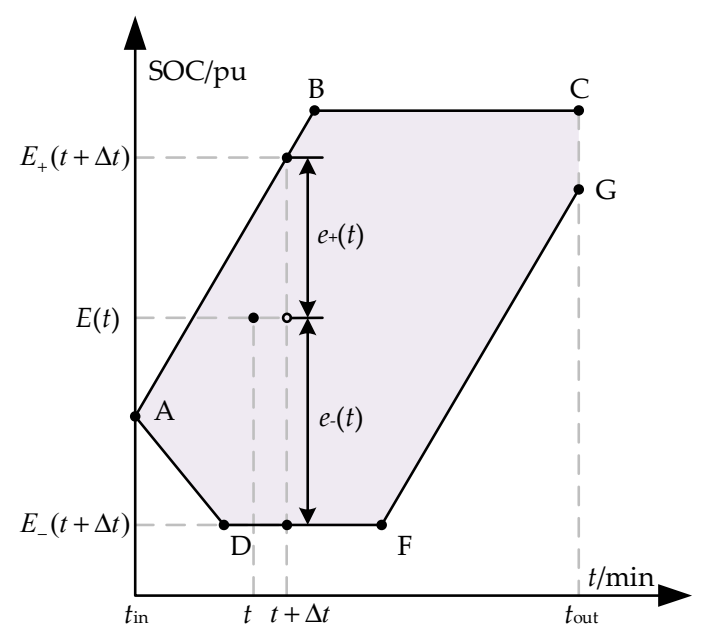

Figure 4. Schematic illustration of charging power range determination method for a controllable EV.

Then the charging power range of EV $i$ at time $t$ can be represented by Equations (11) and (12).

$$
\begin{aligned}
& P_{+}^{i}(t)=\min \left[P_{\mathrm{cmax}}^{i}, e_{+}^{i}(t) \cdot B^{i} / \eta_{\mathrm{c}}^{i}\right] \\
& P_{-}^{i}(t)=\min \left[P_{\mathrm{dmax}}^{i}, e_{-}^{i}(t) \cdot B^{i} \cdot \eta_{\mathrm{d}}^{i}\right]
\end{aligned}
$$

Thus, the charging power range of aggregator $j$ at time $t$ can be obtained, as shown in Equation (13).

$$
\left[-P_{\text {sum }, j}^{-}(t), P_{\text {sum }, j}^{+}(t)\right]=\left[-\sum_{i=1, i \in \Omega_{j}}^{N_{j}} P_{-}^{i}(t), \sum_{i=1, i \in \Omega_{j}}^{N_{j}} P_{+}^{i}(t)\right]
$$

where, $N_{j}$ is the total number of EVs in Aggregator $j, \Omega_{j}$ is the set of EVs participating in power grid scheduling of Aggregator $j,-P_{\text {sum }, j}^{-}(t)$ is the lower boundary of charging power of aggregator $j$, and $P_{\text {sum }, j}^{+}(t)$ is the upper boundary of charging power of aggregator $j$.

Then, the total charging power range of aggregated EVs in the power grid is given by:

$$
\left[-P_{\text {sum }}^{-}(t), P_{\text {sum }}^{+}(t)\right]=\left[-\sum_{j=1}^{K} P_{\text {sum }, j}^{-}(t), \sum_{j=1}^{K} P_{\text {sum }, j}^{+}(t)\right]
$$

where, $K$ is the number of aggregators, $-P_{\text {sum }}^{-}(t)$ and $P_{\text {sum }}^{+}(t)$ are the lower and upper boundary of the total charging power of aggregated EVs in the power grid, respectively. 


\section{Wind-Power Fluctuation Smoothing Strategy of Aggregated EVs in Real Time}

\subsection{Tracking Target of the Total Charging Power of the Aggregated EVs}

The composition of the total load power $P_{\mathrm{S}}$ can be expressed by Equation (15):

$$
P_{\mathrm{S}}=P_{\mathrm{EV}}+P_{0}=P_{\mathrm{EV}}+P_{\mathrm{Non}-\mathrm{EV}}-P_{\mathrm{W}}
$$

where, $P_{\mathrm{EV}}$ is the total charging power of the aggregated EVs in the power grid, $P_{0}$ is the base load power, $P_{\mathrm{Non}-\mathrm{EV}}$ is the non-EV load power, and $P_{\mathrm{W}}$ is wind power.

This paper uses first-order low-pass filtering to determine the real-time power tracking target of the aggregated EVs in the power grid [22,23]. The first-order low-pass filter is one of the most commonly used filtering methods. It has selective passability for low-frequency signals below the cut-off frequency. Its function is to remove the high-frequency components higher than the cut-off frequency in the input composite signal and retain the required low-frequency components. Its mathematical expression can be expressed by Equation (16).

$$
\tau \frac{d u_{y}}{d t}+u_{y}=u_{x}
$$

where, $\tau$ is the time constant of the filter, $u_{\mathrm{x}}$ and $u_{\mathrm{y}}$ are the input and output signal, respectively.

The total load power is filtered on the basis of the first-order low-pass filtering principle mentioned above. Set the total load power before and after filtering at time $t$ are $P_{\mathrm{S}_{-} 0}(t)$ and $P_{\mathrm{S}}(t)$ respectively, where $P_{\mathrm{S}_{-} 0}(t)$ is the sum of the base load power $P_{0}(t)$ and the median of the charging power range of aggregated EVs in the power grid $P_{\text {sum_M }}(t)$, as shown in Equation (17).

$$
P_{\mathrm{S} \_0}(t)=P_{0}(t)+P_{\text {sum } \_\mathrm{M}}(t)=P_{0}(t)+\frac{P_{\text {sum }}^{+}(t)-P_{\text {sum }}^{-}(t)}{2}
$$

Discretize Equation (16) as:

$$
\tau \frac{P_{\mathrm{S}}(t)-P_{\mathrm{S}}(t-\Delta t)}{\Delta t}+P_{\mathrm{S}}(t)=P_{0}(t)+\frac{P_{\text {sum }}^{+}(t)-P_{\text {sum }}^{-}(t)}{2}
$$

From Equation (18), the total load power after filtering $P_{S}(t)$ is given by:

$$
P_{\mathrm{S}}(t)=\frac{\tau}{\tau+\Delta t} P_{\mathrm{S}}(t-\Delta t)+\frac{\Delta t}{\tau+\Delta t}\left(P_{0}(t)+\frac{P_{\mathrm{sum}}^{+}(t)-P_{\text {sum }}^{-}(t)}{2}\right)
$$

It can be known from Equation (19) that when $\tau=0, P_{\mathrm{S}}(t)=P_{\mathrm{S}_{-} 0}(t)$, that is, the filter has no filtering effect on $P_{\mathrm{S}_{-} 0}(t)$. With the increase of filter time constant $\tau, P_{\mathrm{S}}(t)$ gradually approaches $P_{\mathrm{S}}(t-\Delta t)$, and the filtering effect on the total load power becomes more obvious.

From the filtered total load power $P_{\mathrm{S}}(t)$ and real-time base power $P_{0}(t)$, the scheduling power $P_{\mathrm{f}}(t)$ of the aggregated EVs at time $t$ can be calculated as:

$$
P_{\mathrm{f}}(t)=P_{\mathrm{S}}(t)-P_{0}(t)
$$

Then the total charging power of total $\mathrm{EVs} P_{\mathrm{EV}}(t)$ is given by:

$$
P_{\mathrm{EV}}(t)= \begin{cases}-P_{\text {sum }}^{-}(t) & , P_{\mathrm{f}}(t)<-P_{\text {sum }}^{-}(t) \\ P_{\mathrm{f}}(t) & ,-P_{\text {sum }}^{-}(t) \leq P_{\mathrm{f}}(t) \leq P_{\text {sum }}^{+}(t) \\ P_{\text {sum }}^{+}(t) & , P_{\mathrm{f}}(t)>P_{\text {sum }}^{+}(t)\end{cases}
$$




\subsection{The Power Allocation Method of Aggregated EVs}

After calculating the total charging power of the aggregated EVs in the power grid $P_{\mathrm{EV}}(t)$ by the method above, LMCC allocates this power to each aggregator according to the proportion of total charging power. The charging power of the aggregator $j$ can be given by:

$$
P_{\mathrm{A}, j}(t)= \begin{cases}-P_{\text {sum }, j}^{-}(t) & , P_{\mathrm{f}}(t)<-P_{\text {sum }}^{-}(t) \\ \frac{\left(P_{\mathrm{Ev}}(t)+P_{\text {sum }}^{-}(t)\right)\left(P_{\text {sum }, j}^{+}(t)+P_{\text {sum }, j}^{-}(t)\right)}{P_{\text {sum }}^{+}(t)+P_{\text {sum }}^{-}(t)}-P_{\text {sum }, j}^{-}(t) & ,-P_{\text {sum }}^{-}(t) \leq P_{\mathrm{f}}(t) \leq P_{\text {sum }}^{+}(t) \\ P_{\text {sum }, j}^{+}(t) & , P_{\mathrm{f}}(t)>P_{\text {sum }}^{+}(t)\end{cases}
$$

Then, aggregator $j$ allocates its total charging power according to the controllable degree of each $\mathrm{EV}$ in the aggregator. This paper defines the controllable coefficient of EV $i Q^{i}(t)$ at time $t$ as:

$$
\begin{gathered}
Q^{i}(t)=\frac{T_{\mathrm{cmin} *}^{i}(t+\Delta t)}{t_{\text {out }}^{i}-t} \\
T_{\mathrm{cmin} *}^{i}(t+\Delta t)=\frac{\left(E_{\text {out }}^{i}-E^{i}(t+\Delta t)\right)}{\eta_{\mathrm{c}} P_{\mathrm{cmax}}}
\end{gathered}
$$

where $T_{\text {cmin* }}^{i}(t+\Delta t)$ is the shortest charging duration of EV $i$ at time $t+\Delta t$.

The smaller the total controllable coefficient of the EVs participating in power grid scheduling is, the larger the charging and discharging margin of the aggregated EVs as energy storage, and the stronger the ability to smooth the wind power fluctuations. In this paper, the total charging power of the aggregator is allocated to minimize the sum of the controllable coefficients of aggregated EVs. The optimization objective is given by:

$$
\min \sum_{i=1, i \in \Omega_{j}}^{N_{j}} Q^{i}
$$

where, $N_{j}$ is the number of EVs in aggregator $j$.

The constraints of this model are as follows:

$$
\begin{gathered}
-P_{-}^{i}(t) \leq P^{i}(t) \leq P_{+}^{i}(t) \quad \forall i \in \Omega_{j} \\
P_{\mathrm{EV}}(t)=\sum_{i=1, i \in \Omega_{j}}^{N_{j}} P^{i}(t) \\
E^{i}(t+\Delta t)=E^{i}(t)+\Delta E^{i}(t) \quad \forall i \in \Omega_{j} \\
\Delta E^{i}(t)= \begin{cases}P^{i}(t) / \eta_{\mathrm{d}}^{i} / B^{i} & ,-P_{-}^{i}(t) \leq P^{i}(t) \leq 0 \quad \forall i \in \Omega_{j} \\
P^{i}(t) \cdot \eta_{\mathrm{c}}^{i} / B^{i} & , 0<P^{i}(t) \leq P_{+}^{i}(t)\end{cases}
\end{gathered}
$$

where, $P^{i}(t)$ is the charging power of EV $i$ at time $t$, and $\Delta E^{i}(t)$ is the SOC variation of EV $i$ from time $t$ to time $t+\Delta t$. Equation (26) indicates that the charging power of each EV must meet its charging power boundary condition; Equation (27) indicates that the total charging power of the aggregator is equal to the sum of the charging power of each EV; Equations (28) and (29) indicate the SOC update of each EV.

Perform piecewise linearization on Equation (29), and introduce $0-1$ type variables and continuous variables $w_{1}^{i}, w_{2}^{i}$ and $w_{3}^{i}$, and set points $b_{1}=-P_{-}^{i}(t), b_{2}=0$, and $b_{3}=P_{+}^{i}(t)$. The non-linear constraint (29) is transformed into a linear constraint (30). 


$$
\left\{\begin{array}{l}
w_{1}^{i}+w_{2}^{i}+w_{3}^{i}=1 \\
z_{1}^{i}+z_{2}^{i}=1 \\
w_{1}^{i} \leq z_{1}^{i} \\
w_{2}^{i} \leq z_{1}^{i}+z_{2}^{i} \\
w_{3}^{i} \leq z_{2}^{i} \\
P^{i}(t)=\sum_{n=1}^{3} w_{n}^{i} b_{n} \\
\Delta E^{i}(t)=\left.\sum_{n=1}^{3} w_{n}^{i} \Delta E^{i}(t)\right|_{P^{i}(t)=b_{n}}
\end{array} \quad \forall i\right.
$$

Equations (25)-(27) and (30) constitute a MILP problem, which can be solved easily by an MILP solver, such as CPLEX and Gurobi [24]. We can obtain the charging power of each EV after solving this MILP problem.

\subsection{Real-Time Control Flow for Aggregated EVs}

The flowchart of the real-time control strategy for aggregated EVs to smooth fluctuation of wind power output in this paper is shown in Figure 5.

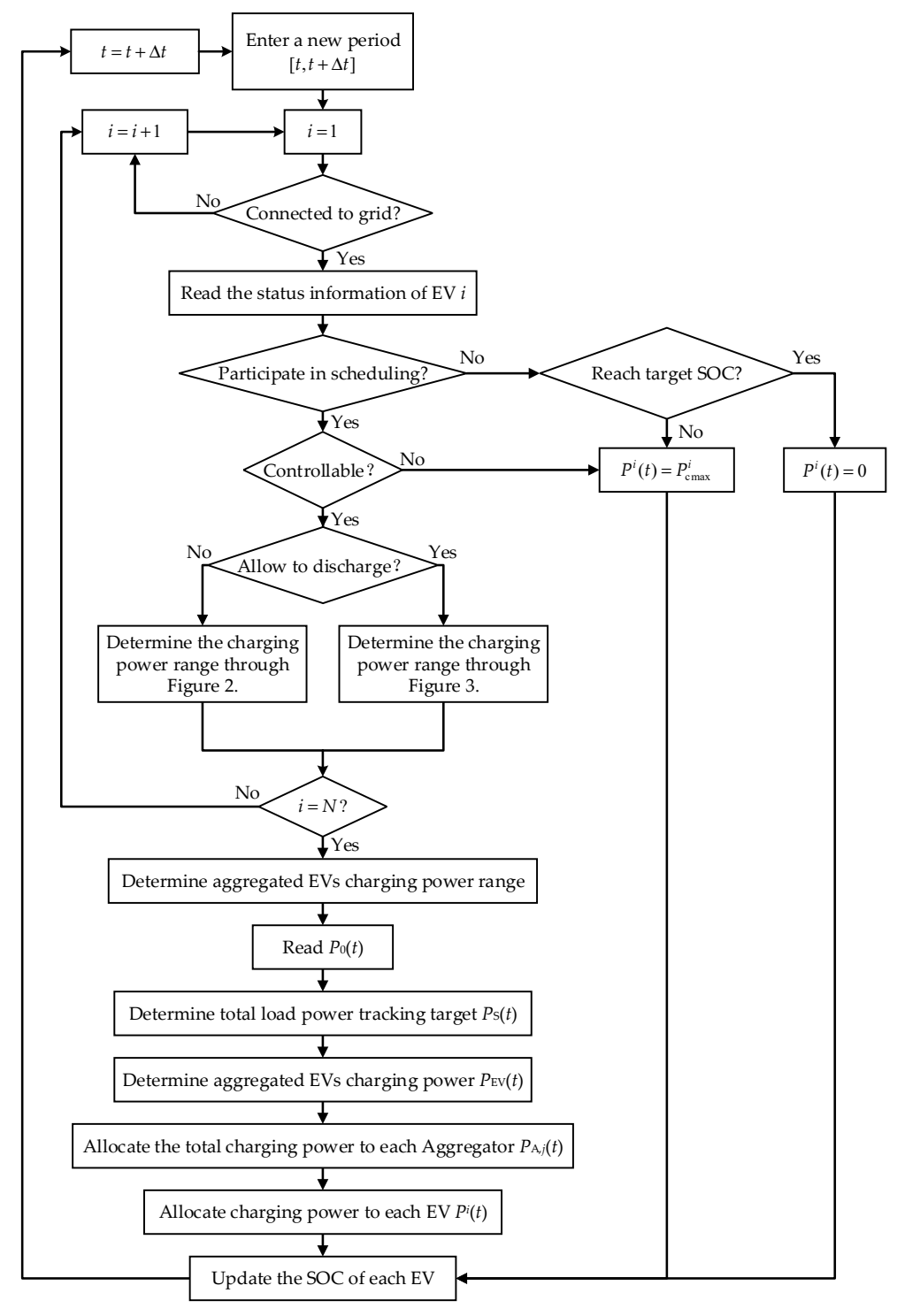

Figure 5. Control flowchart of aggregated EVs to smooth fluctuation. 


\section{Case Analysis}

\subsection{Case Data}

In order to verify the effectiveness of the real-time control strategy for aggregated EVs proposed in this paper, a regional distribution system is taken as an example for simulation analysis. The simulation time interval is $1 \mathrm{~d}$ (0:00-24:00) and the simulation sampling time is $\Delta t=5 \mathrm{~min}$. The non-EV load power curve is shown in Figure 6, and the output power curve of the wind farm is shown in Figure 7. Assume that the number of EVs in this area is 150, of which $10 \%$ do not participate in scheduling, $40 \%$ are involved in scheduling but not in V2G, and 50\% are involved in both processes. The parameter settings of the aggregated EVs are the same. Among them, the battery capacity is $60 \mathrm{kWh}$, and the maximum charging and discharging power are $12 \mathrm{~kW}$ and $5 \mathrm{~kW}$, respectively, with their efficiencies both 0.95 . The filter time constant is set as $45 \mathrm{~min}$. This paper references [25], performs data mining on the 2017 National Household Travel Survey (2017NHTS) data, and then carries out trip chain simulation based on the Monte-Carlo method to obtain the charging demand of the aggregated EVs. A brief introduction to the simulation method and the simulation results are shown in Appendix A.

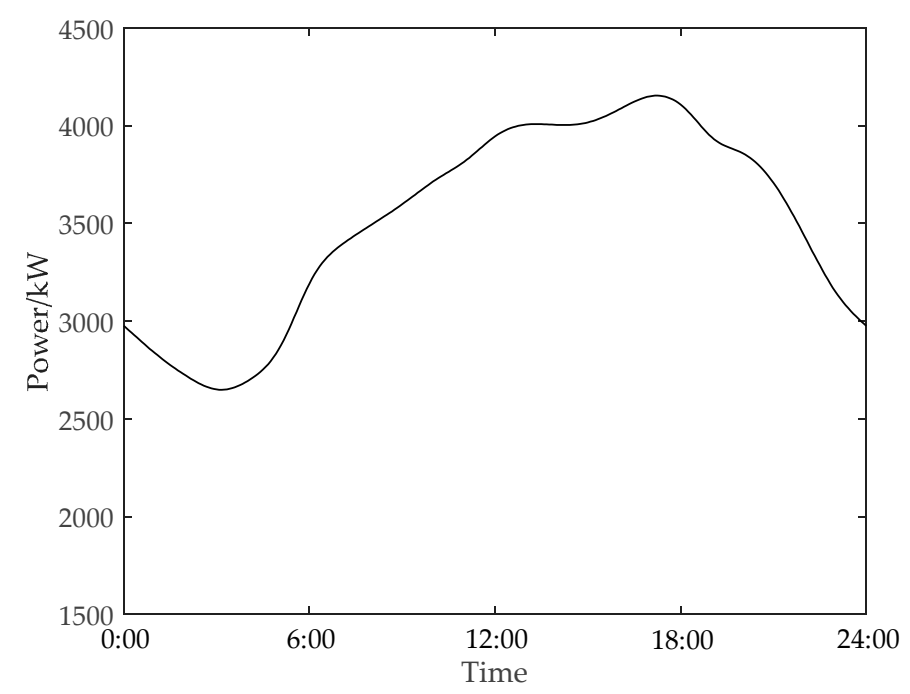

Figure 6. Non-EV load curve.

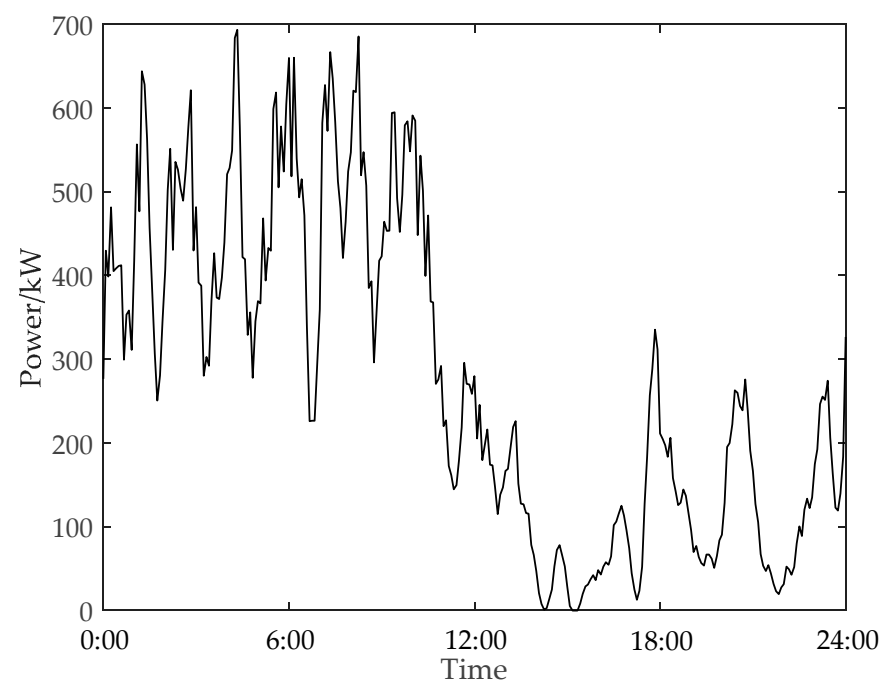

Figure 7. Wind-power output curve. 
In order to quantitatively analyze the smoothing effect of wind-power fluctuation, the fluctuation rate of total load power $\Delta P_{\mathrm{S}} \%$ at time $t_{0}$ is defined as [26]:

$$
\begin{gathered}
\Delta P_{\mathrm{S}} \%=\frac{\sigma}{\lambda} \\
\sigma=\sqrt{\frac{\Delta t}{T_{0}} \sum_{t=t_{0}}^{t_{0}-T_{0}}\left[P_{\mathrm{S}}(t)-P_{\mathrm{S}_{-} T_{0} \_\mathrm{av}}\right]^{2}} \\
\lambda=\sqrt[T_{0} / \Delta t]{\prod_{t=t_{0}}^{t_{0_{0}}-T_{0}} P_{\mathrm{S}}(t)}
\end{gathered}
$$

where, $\sigma$ and $\lambda$ are the standard deviation and the geometric mean of the total load power respectively, $T_{0}$ is the calculation time scale of the fluctuation rate, and $P_{\mathrm{S}_{-} T_{0 \_}}$av is the average total load power during the period from $t_{0}-T_{0}$ to $t_{0}$.

\subsection{Results Analysis}

\subsubsection{Results of Real-Time Control Strategy for Aggregated EVs to Smooth Wind Power Fluctuation}

Assume that the aggregated EVs do not accept grid scheduling, and all EVs charge at the rated maximum charging power as soon as they are connected to the grid, until the charging demand is met or they leave. The total load curve in this disorderly charging mode is shown in red curve in Figure 8, in which the gray curve represents the base load curve and the blue curve represents the total load curve under the real-time control strategy proposed in this paper. The fluctuation rates of the total load power under the two charging modes are shown in Figure 9, where the calculation time scale of the fluctuation rate is $30 \mathrm{~min}$. The number of EVs participating in grid scheduling in each time period is shown in Figure 10. The scheduling power and real-time response of total charging power of aggregated EVs are shown in Figure 11.

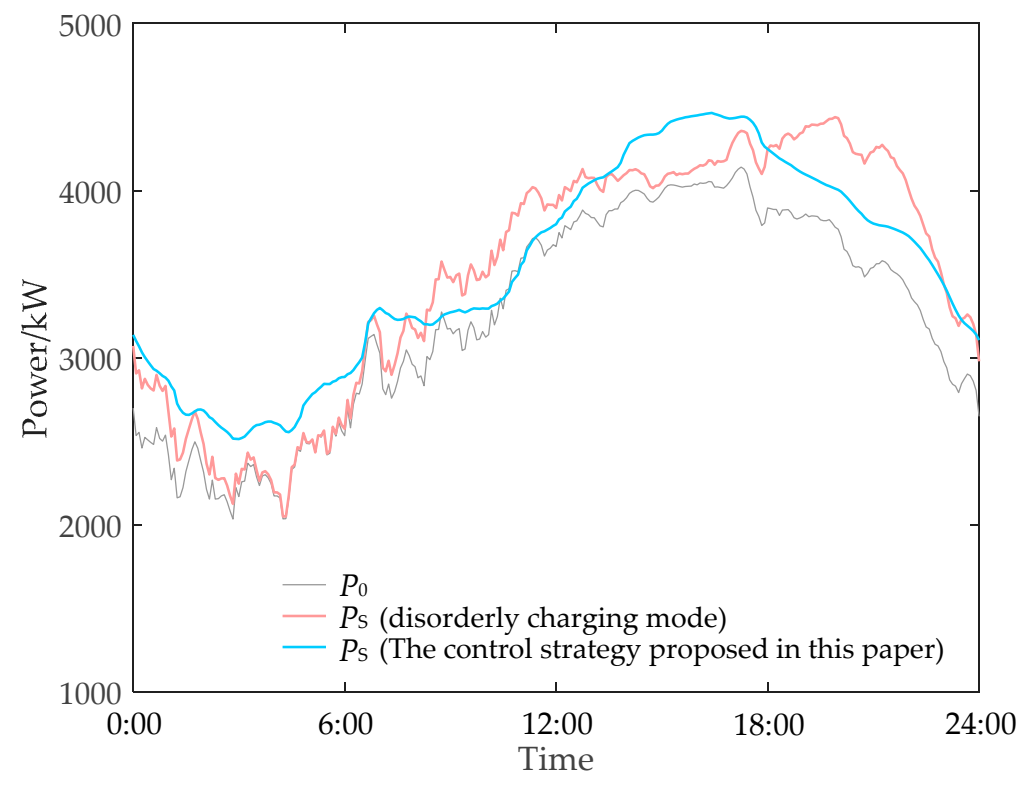

Figure 8. Total load curve. 


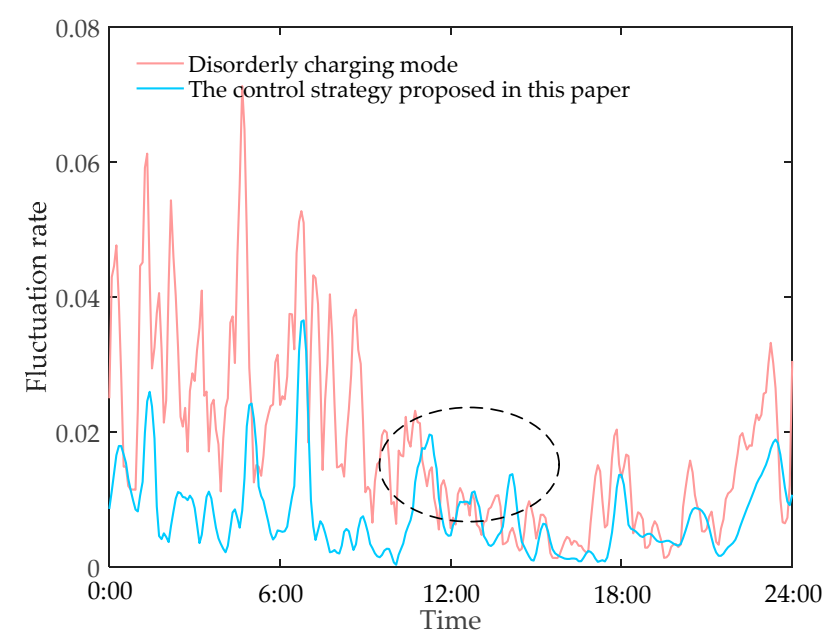

Figure 9. Total load power fluctuation rate.

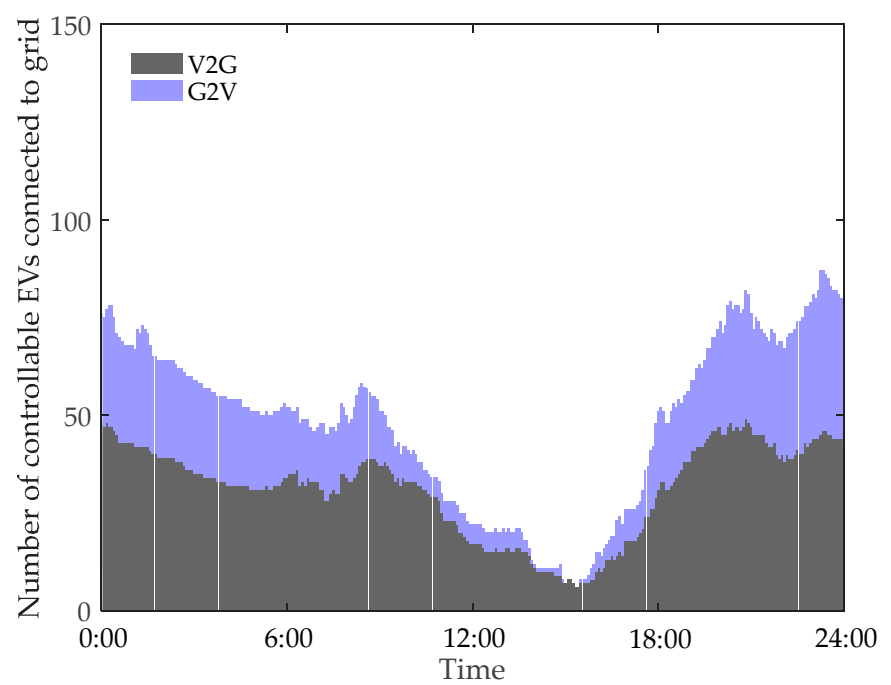

Figure 10. Number of controllable EVs participating in grid scheduling.

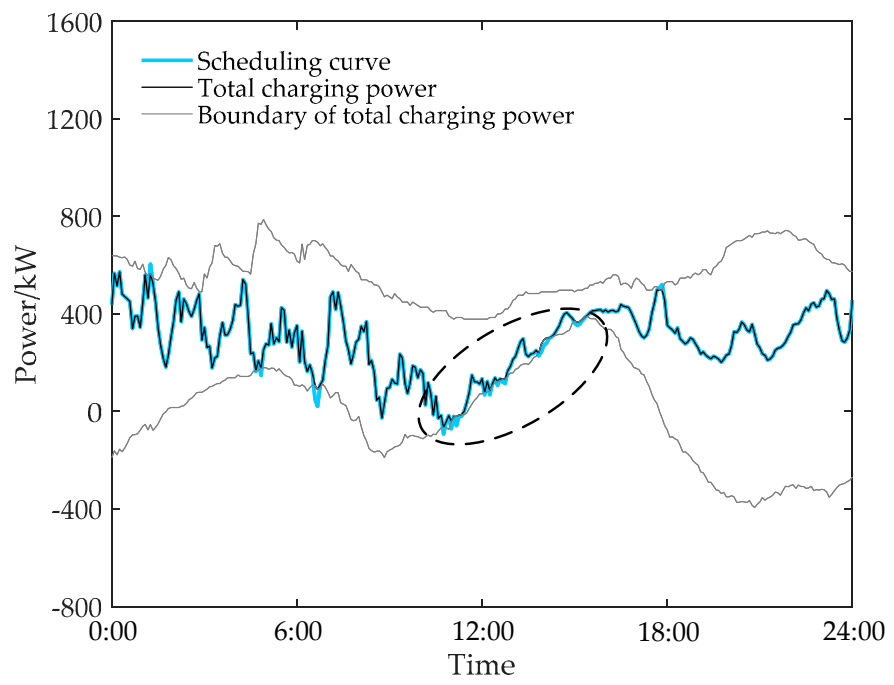

Figure 11. Scheduling power and total charging power.

It can be seen from Figures 8 and 9 that under the real-time control strategy proposed in this paper, the fluctuation rate of the total load is greatly decreased, and the total load curve is smoother, 
as well as the peak-valley difference of the total load being reduced. While in the disorderly charging mode, the average total load power fluctuation rate is 0.0179 , and the maximum and minimum total load power are $4440 \mathrm{~kW}$ and $2050 \mathrm{~kW}$, respectively; the real-time control strategy proposed in this paper can make the average total load power fluctuation rate only 0.0078 , the maximum $4464 \mathrm{~kW}$ and minimum $2513 \mathrm{~kW}$. The total load power fluctuation rate and peak-valley difference can be reduced by $56.4 \%$ and $18.4 \%$, respectively.

As can be seen from Figures 10 and 11, during the periods of 0: 00-6: 00 and 20: 00-24: 00, there are a large number of controllable EVs participating in grid scheduling. At this time, the total charging power range of aggregated EVs is wide, and the ability to smooth total load power fluctuation is strong. The scheduling power issued by the LMCC to the aggregators is within the boundary of the total charging power, so the aggregated EVs can respond to the scheduling power in real time. During the period from 10:00 to 18:00, the number of controllable EVs participating in grid scheduling is small. At this time, the total charging power range of aggregated EVs is narrow, and the ability to smooth the total load power fluctuation is relatively weak. The scheduling power is beyond the total charging power boundary so that the aggregated EVs will not be able to track the scheduling power (as shown in the black circle in Figure 11), and the total load power fluctuation rate at the corresponding moment increases greatly (as shown in the black circle in Figure 9).

\subsubsection{Analysis of the Influence of the Filter Time Constant on Smoothing Effect}

The simulation is performed in the way of changing the filter time constant and keeping other parameters unchanged. The fluctuation rate of the total load curve under the real-time control strategy of the aggregated EVs obtained by simulation is shown in Figure 12, and the scheduling power and the total charging power of aggregated EVs are shown in Figure 13. Some quantitative results are shown in Table 1.

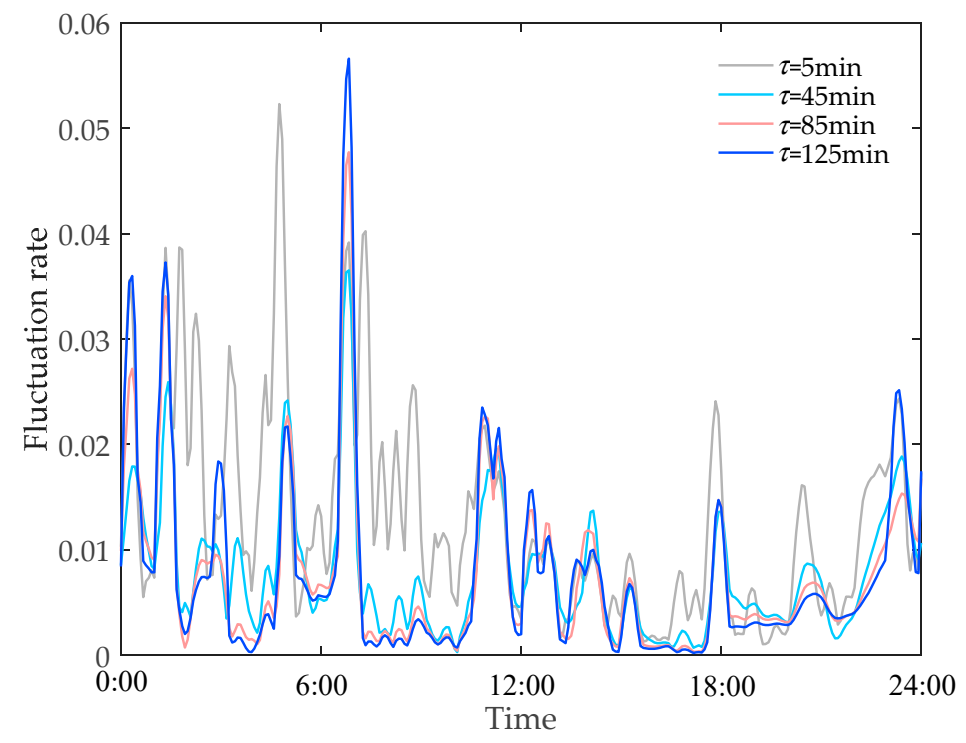

Figure 12. Total load power fluctuation rate under different filter time constants.

Table 1. Simulation results under different filter time constants.

\begin{tabular}{ccc}
\hline$\tau(\mathbf{s})$ & Average Power Fluctuation Rate & Number of Scheduling Power Untracked \\
\hline $5 \mathrm{~min}$ & 0.01302 & 0 \\
$45 \mathrm{~min}$ & 0.00742 & 27 \\
$85 \mathrm{~min}$ & 0.00756 & 43 \\
$125 \mathrm{~min}$ & 0.00785 & 54 \\
\hline
\end{tabular}



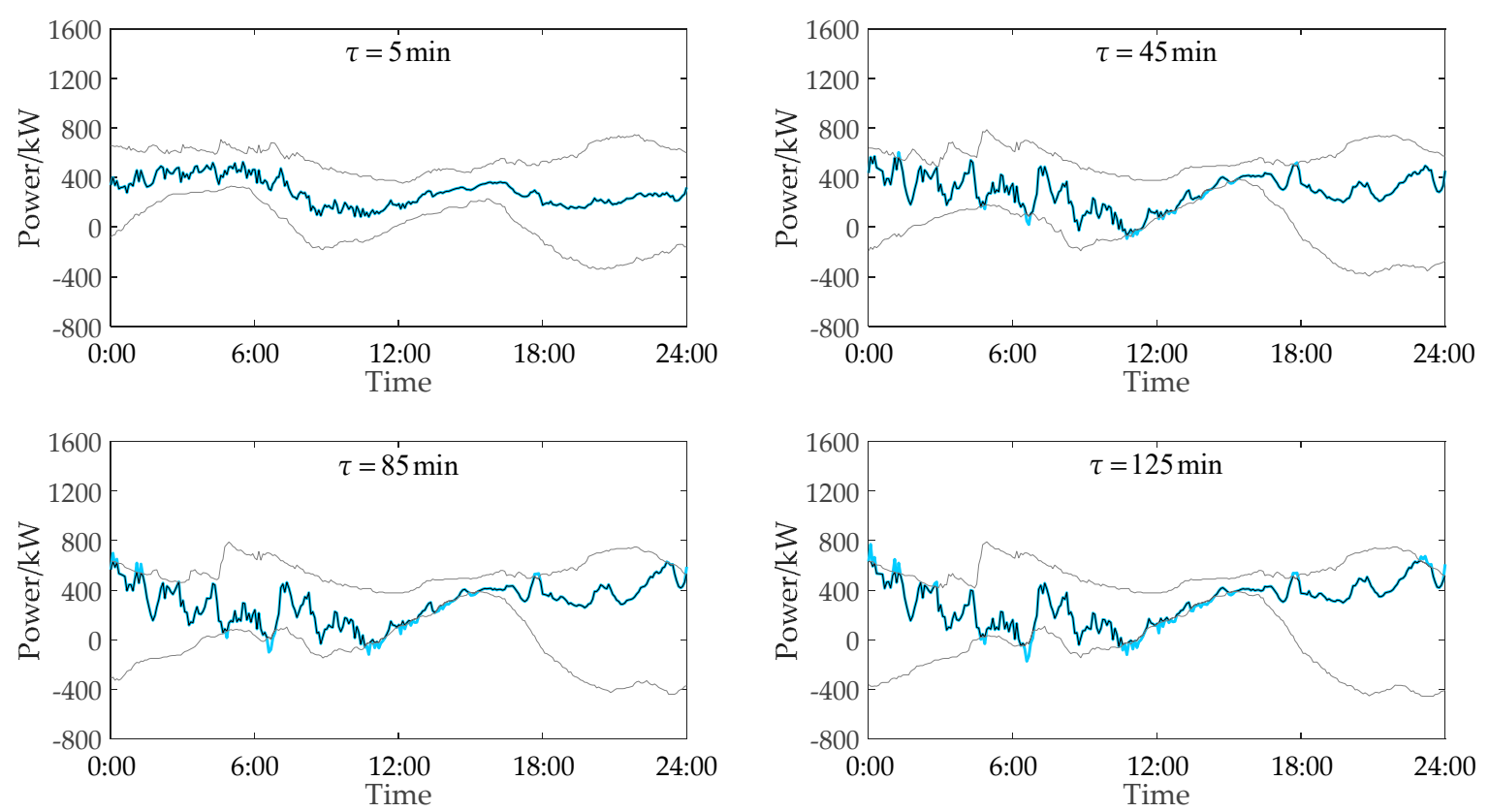

Figure 13. Total charging power of aggregated EVs under different filter time constants.

From the simulation results, it can be seen that when the filter time constant $\tau$ is small, the total load fluctuates greatly. This is because when $\tau$ is small, the filter cut-off frequency is large, and the fluctuation component is small. With the gradual increase of $\tau$, the filter cut-off frequency decreases, the fluctuation component of filtered total load power increases, and the fluctuation of total load power decreases. The total load power fluctuation rate at $\tau=45 \mathrm{~min}$ is significantly smaller than fluctuation rate at $\tau=5 \mathrm{~min}$. However, as $\tau$ continues to increase, the fluctuation of the scheduling power of the aggregated EVs also increases, and the probability of failure to follow the scheduling power increases due to the influence of the upper and lower boundaries of the total charging power. It can be seen from Figure 13 that at $\tau=85 \mathrm{~min}$ and $\tau=125 \mathrm{~min}$, during the period of 10:00-18:00, as a result of the small number of EVs participating in scheduling in the grid, the controllable range of the total charging power of the aggregated EV becomes small and the probability of scheduling power not being tracked is greatly increased.

5.2.3. Analysis of the Influence of the Proportion of EVs in Vehicle-to-Grid (V2G) Mode on Smoothing Effect

The simulation is performed in the way of changing the proportion of EVs participating in V2G and keeping other parameters unchanged. The average total load power fluctuation rate under different proportions of EVs in V2G is shown in Figure 14. It can be seen from the results that, as the proportion of EVs in $\mathrm{V} 2 \mathrm{G}$ increases, the average fluctuation rate of the total load power decreases. This is because the EVs participating in V2G have better power regulation performance than those that participate in G2V.

At 5:20 and 5:30, the wind power output suddenly dropped by $143.2 \mathrm{~kW}$ and $128 \mathrm{~kW}$, respectively, as shown in Figure 15. The total charging power responding to the aggregated EVs in V2G and G2V modes are shown in Figure 16. In the V2G mode, the total charging power of aggregated EVs can track the scheduling power, while in the G2V mode, the aggregated EVs cannot accurately do so. The EVs in V2G mode have better power regulation performance than in G2V mode, and can provide stronger power support for the distribution system in emergency situations. 


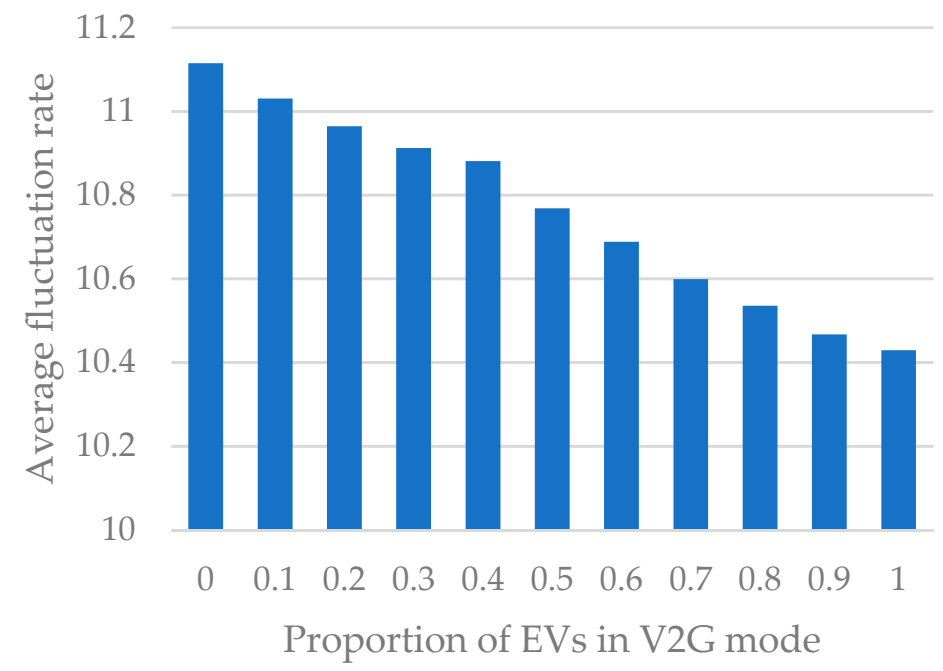

Figure 14. The average fluctuation rate of total load under different proportions of the EVs in V2G mode.

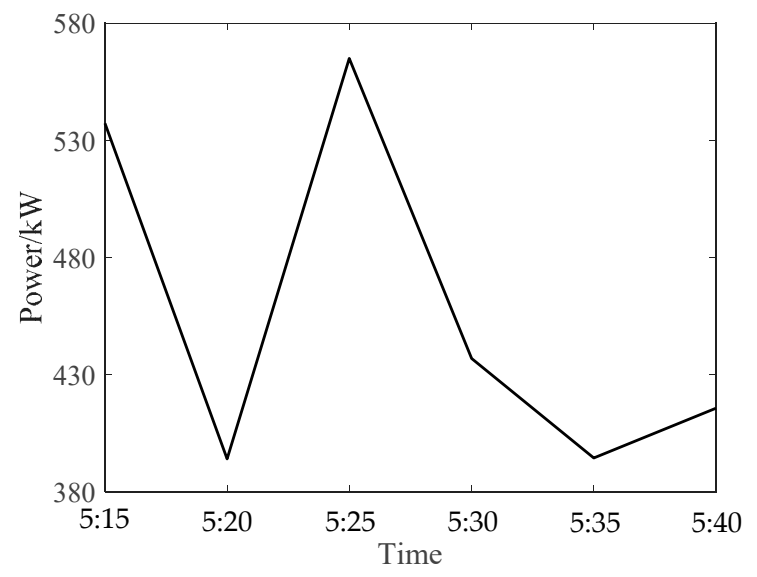

Figure 15. Sudden drop in wind energy output.

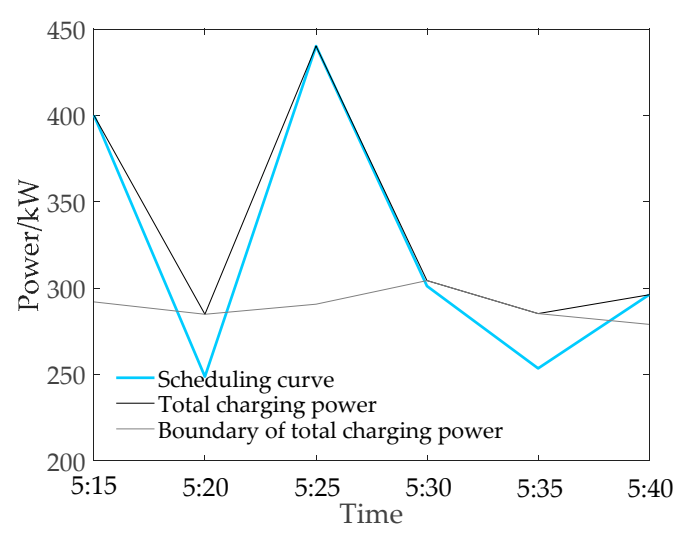

(a)

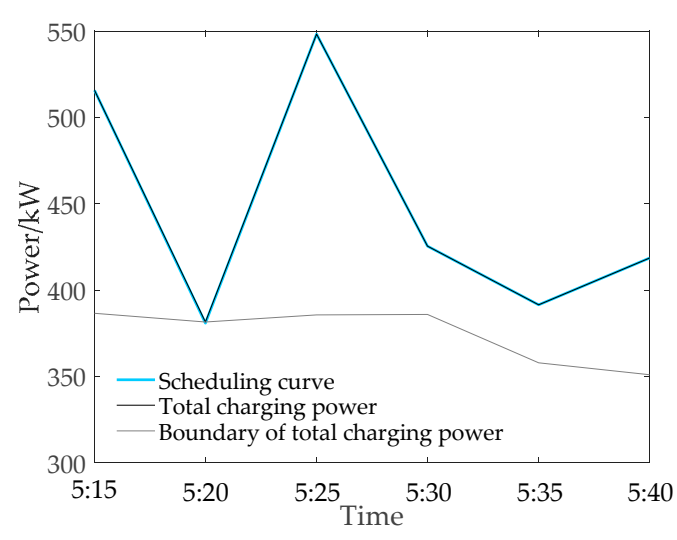

(b)

Figure 16. Comparison of results responding to the EVs in V2G mode and G2V mode in emergency situations: (a) G2V mode; (b) V2G mode.

\section{Conclusions}

It is both an opportunity and a challenge to connect large-scale EVs to the power grid. Because of the flexibility and controllability of the charging load of EVs, reasonable and orderly control of their charging/discharging processes can achieve friendly interaction with the distribution system. 
This paper proposes a real-time control strategy for the aggregated EVs to smooth fluctuation of wind power output. The simulation results show that:

(1) On the premise of meeting the charging needs of EV users, the real-time control strategy for aggregated EVs proposed in this paper does not need to forecast the load and wind-power output. Real-time smoothing of wind-power fluctuation can be achieved through first-order low-pass filtering algorithms, which can smooth the total load curve, and can reduce the peak-valley difference of total load power to ensure the safe and reliable operation of the distribution system.

(2) The time constant of the filter will affect the smoothing effect of the aggregated EVs on total load power fluctuations. If the time constant of the filter is set too small, the aggregated EVs will filter out only a small part of the load fluctuation component in the area, and the smoothing effect will be poor; if the time constant of the filter is set too large, the probability of the total charging power of the aggregated EVs failing to track the dispatching power will increase.

(3) Compared with the EVs in G2V mode, those in V2G mode have better power regulation performance, which can provide power support for the distribution system in emergency situations.

Author Contributions: Y.Z., Y.T., and Z.Y. designed the study; P.G. collected the simulation data; Z.Y. and Y.T. carried out the simulation and analyses; Y.T., Z.W. and Z.Y. wrote the manuscript; and R.X. and Y.Z. reviewed and edited the manuscript. All authors have read and agreed to the published version of the manuscript.

Funding: This research was supported by Open Fund of Operation and Control of Renewable Energy \& Storage Systems (China Electric Power Research Institute) (No. NYB51201801553).

Conflicts of Interest: The authors declare no conflict of interest.

\section{Appendix A}

\section{Charging Demand Simulation Method Based on Trip Chain}

A trip chain is a schedule of activities in time and space. As shown in Figure A1, the variables of a trip chain can be divided into two categories:

Time variables:

(1) $t_{\text {in } \_i}$ : arrival time of the $i$-th trip destination;

(2) $t_{\text {out } \_} i$ leaving time of the $i$ -

(3) th destination;

(4) $T_{\mathrm{x}(i-1, i)}$ : the driving duration between the (i-1)-th destination and $i$-th destination;

(5) $T_{\mathrm{p}_{i} i}$ : the parking duration at the $i$-th destination.

Space variables:

(1) $\left\{D_{k} \mid k=1,2, \ldots, U\right\}(\mathrm{U}$ is the total number of destination types): destination type collection, and type (i) $=D_{k}$ indicates that the type of the $i$-th destination is $D_{k}$;

(2) $d_{(i-1, i)}$ : the trip mileage between the $(i-1)$-th destination and $i$-th destination.

\section{Time chain}

Space chain

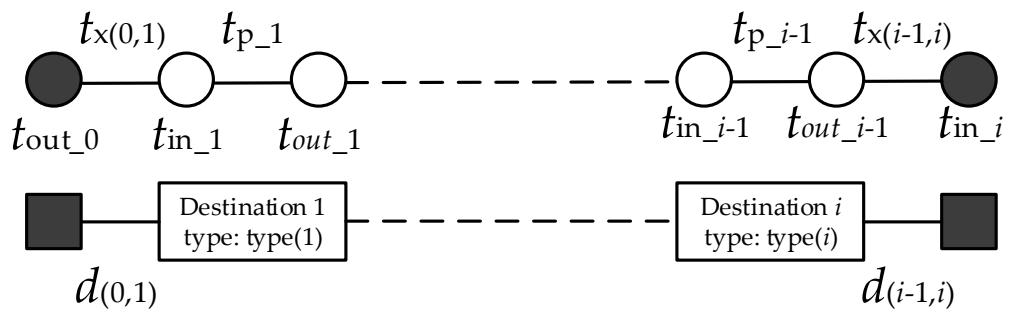

Figure A1. Schematic diagram of trip chain.

The probability distributions of all the time and space variables can be obtained in discrete or continuous forms, which will be inputs of Monte Carlo simulation. Due to the mutual influence of 
variables of the space chain and time chain, the Monte Carlo method is used repeatedly and only one variable's sample in one dimension is extracted in turn, while the relationships of these variables are reflected by the probability distributions. The flowchart of trip chain simulation is shown in Figure A2. The simulation results based on NHTS data is shown in Table A1.

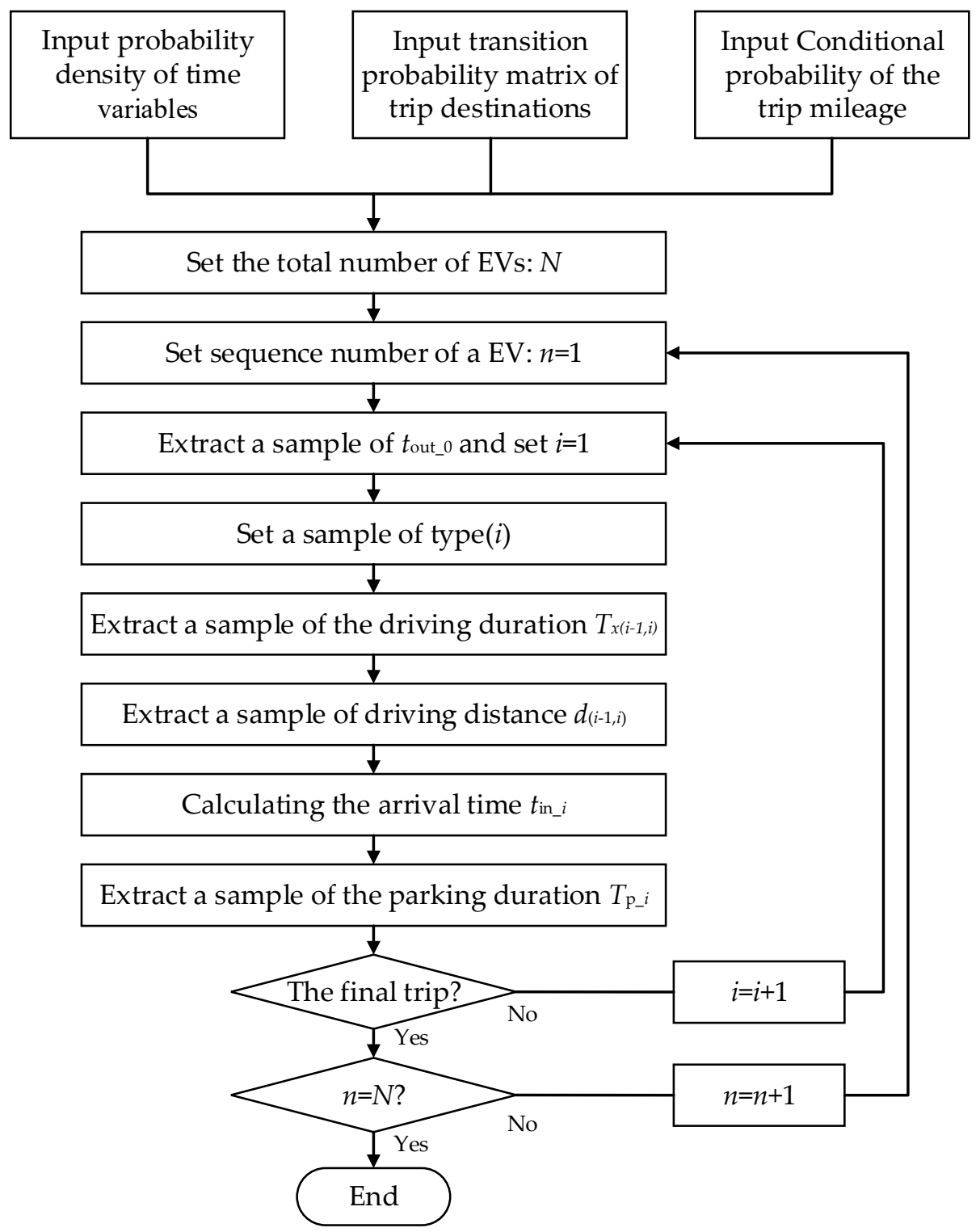

Figure A2. Flowchart of trip chain simulation.

Table A1. Simulation results of daily charging demand.

\begin{tabular}{|c|c|c|c|c|c|c|c|c|c|}
\hline \multirow[b]{2}{*}{ No. } & \multicolumn{2}{|c|}{ First Charge } & \multicolumn{2}{|c|}{ Second Charge } & \multirow[b]{2}{*}{ No. } & \multicolumn{2}{|c|}{ First Charge } & \multicolumn{2}{|c|}{ Second Charge } \\
\hline & $\begin{array}{l}\text { On-Grid } \\
\text { Time }\end{array}$ & $\begin{array}{l}\text { Off-Grid } \\
\text { Time }\end{array}$ & $\begin{array}{l}\text { On-Grid } \\
\text { Time }\end{array}$ & $\begin{array}{c}\text { Off-Grid } \\
\text { Time }\end{array}$ & & $\begin{array}{l}\text { On-Grid } \\
\text { Time }\end{array}$ & $\begin{array}{c}\text { Off-Grid } \\
\text { Time }\end{array}$ & $\begin{array}{l}\text { On-Grid } \\
\text { Time }\end{array}$ & $\begin{array}{c}\text { Off-Grid } \\
\text { Time }\end{array}$ \\
\hline 1 & $6: 55$ & $16: 38$ & $19: 50$ & $\downarrow 7: 36$ & 76 & $8: 32$ & $17: 20$ & & \\
\hline 2 & $\uparrow 17: 28$ & $7: 15$ & $20: 49$ & $\downarrow 8: 32$ & 77 & $\uparrow 16: 59$ & $8: 47$ & $19: 10$ & $\downarrow 7: 57$ \\
\hline 3 & $\uparrow 15: 50$ & $8: 26$ & $18: 51$ & $\downarrow 7: 41$ & 78 & $\uparrow 18: 58$ & 7:57 & 18:18 & $\downarrow 8: 50$ \\
\hline 4 & $\uparrow 15: 48$ & $7: 22$ & $19: 15$ & $\downarrow 7: 15$ & 79 & $\uparrow 16: 31$ & $7: 37$ & $17: 32$ & $\downarrow 6: 18$ \\
\hline
\end{tabular}


Table A1. Cont.

\begin{tabular}{|c|c|c|c|c|c|c|c|c|c|}
\hline \multirow[b]{2}{*}{ No. } & \multicolumn{2}{|c|}{ First Charge } & \multicolumn{2}{|c|}{ Second Charge } & \multirow[b]{2}{*}{ No. } & \multicolumn{2}{|c|}{ First Charge } & \multicolumn{2}{|c|}{ Second Charge } \\
\hline & $\begin{array}{l}\text { On-Grid } \\
\text { Time }\end{array}$ & $\begin{array}{l}\text { Off-Grid } \\
\text { Time }\end{array}$ & $\begin{array}{l}\text { On-Grid } \\
\text { Time }\end{array}$ & $\begin{array}{l}\text { Off-Grid } \\
\text { Time }\end{array}$ & & $\begin{array}{l}\text { On-Grid } \\
\text { Time }\end{array}$ & $\begin{array}{l}\text { Off-Grid } \\
\text { Time }\end{array}$ & $\begin{array}{l}\text { On-Grid } \\
\text { Time }\end{array}$ & $\begin{array}{c}\text { Off-Grid } \\
\text { Time }\end{array}$ \\
\hline 5 & $\uparrow 21: 01$ & $7: 53$ & 21:00 & $\downarrow 7: 14$ & 80 & $\uparrow 19: 20$ & $6: 17$ & $20: 53$ & $\downarrow 7: 38$ \\
\hline 6 & $\uparrow 17: 40$ & $9: 22$ & $13: 13$ & $20: 49$ & 81 & $\uparrow 18: 32$ & $7: 11$ & & \\
\hline 7 & $17: 36$ & $\downarrow 8: 19$ & & & 82 & $\uparrow 19: 12$ & $6: 34$ & 19:06 & $\downarrow 6: 54$ \\
\hline 8 & $17: 49$ & $\downarrow 7: 20$ & & & 83 & $\uparrow 19: 54$ & 9:08 & $17: 31$ & $\downarrow 9: 02$ \\
\hline 9 & $\uparrow 20: 49$ & $8: 12$ & $18: 14$ & $\downarrow 8: 28$ & 84 & $\uparrow 19: 48$ & $5: 48$ & & \\
\hline 10 & 12:55 & $15: 47$ & $20: 30$ & $\downarrow 7: 29$ & 85 & $\uparrow 15: 40$ & $5: 48$ & $18: 17$ & $\downarrow 7: 59$ \\
\hline 11 & 11:18 & $17: 16$ & & & 86 & $\uparrow 19: 51$ & 9:11 & $20: 29$ & $\downarrow 7: 13$ \\
\hline 12 & 21:05 & $\downarrow 7: 30$ & & & 87 & $\uparrow 19: 21$ & $6: 11$ & & \\
\hline 13 & $\uparrow 16: 22$ & $8: 02$ & $17: 29$ & $\downarrow 8: 11$ & 88 & $8: 31$ & $13: 40$ & & \\
\hline 14 & $17: 05$ & $\downarrow 7: 44$ & & & 89 & $\uparrow 15: 21$ & $8: 29$ & $17: 00$ & $\downarrow 5: 38$ \\
\hline 15 & $13: 35$ & 19:01 & & & 90 & $\uparrow 15: 39$ & $7: 35$ & $21: 14$ & $\downarrow 5: 54$ \\
\hline 16 & $16: 47$ & $\downarrow 6: 58$ & & & 91 & $9: 48$ & $15: 58$ & & \\
\hline 17 & 9:09 & $17: 17$ & $19: 54$ & $\downarrow 5: 28$ & 92 & $8: 25$ & $14: 40$ & & \\
\hline 18 & $15: 59$ & $\downarrow 10: 16$ & & & 93 & $\uparrow 17: 43$ & 8:09 & 22:05 & $\downarrow 5: 38$ \\
\hline 19 & $\uparrow 17: 50$ & $9: 14$ & $16: 44$ & $\downarrow 8: 21$ & 94 & $20: 52$ & $\downarrow 7: 04$ & & \\
\hline 20 & $8: 50$ & $16: 18$ & & & 95 & 9:00 & $14: 21$ & & \\
\hline 21 & $13: 15$ & $19: 03$ & & & 96 & $20: 29$ & $\downarrow 8: 34$ & & \\
\hline 22 & $\uparrow 17: 07$ & $5: 12$ & 13:06 & $17: 53$ & 97 & $\uparrow 20: 20$ & $4: 15$ & & \\
\hline 23 & $\uparrow 20: 02$ & $5: 50$ & & & 98 & $8: 43$ & $18: 23$ & & \\
\hline 24 & $\uparrow 18: 40$ & $9: 26$ & $16: 01$ & $\downarrow 7: 35$ & 99 & $\uparrow 18: 14$ & $6: 50$ & $16: 59$ & $\downarrow 10: 19$ \\
\hline 25 & $10: 29$ & $19: 32$ & & & 100 & $\uparrow 18: 23$ & $8: 52$ & $17: 02$ & $\downarrow 6: 06$ \\
\hline 26 & $\uparrow 17: 25$ & $8: 38$ & $18: 55$ & $\downarrow 9: 45$ & 101 & $\uparrow 17: 31$ & $5: 33$ & $18: 11$ & $\downarrow 9: 59$ \\
\hline 27 & $\uparrow 16: 18$ & 9:57 & $17: 32$ & $\downarrow 9: 23$ & 102 & 19:44 & $\downarrow 7: 19$ & & \\
\hline 28 & $9: 37$ & $14: 21$ & & & 103 & $5: 47$ & $14: 42$ & & \\
\hline 29 & $7: 17$ & $16: 45$ & & & 104 & $9: 31$ & $15: 06$ & & \\
\hline 30 & $\uparrow 17: 08$ & 7:56 & $18: 16$ & $\downarrow 9: 52$ & 105 & $\uparrow 18: 29$ & 8:05 & $19: 23$ & $\downarrow 7: 00$ \\
\hline 31 & $\uparrow 19: 04$ & $4: 41$ & 19:01 & $\downarrow 10: 52$ & 106 & $\uparrow 18: 07$ & $10: 14$ & & \\
\hline 32 & $\uparrow 19: 25$ & $6: 44$ & 13:10 & $17: 53$ & 107 & $\uparrow 17: 29$ & $10: 53$ & 19:05 & $\downarrow 10: 52$ \\
\hline 33 & $\uparrow 18: 17$ & $6: 13$ & $19: 27$ & $\downarrow 6: 22$ & 108 & $\uparrow 18: 57$ & $8: 31$ & $18: 11$ & $\downarrow 7: 18$ \\
\hline 34 & $7: 59$ & $16: 19$ & & & 109 & $\uparrow 22: 53$ & $7: 29$ & $17: 58$ & $\downarrow 7: 40$ \\
\hline 35 & 13:07 & $20: 31$ & & & 110 & 19:36 & $\downarrow 8: 16$ & & \\
\hline 36 & $\uparrow 18: 27$ & $8: 43$ & $16: 37$ & $\downarrow 7: 09$ & 111 & $8: 17$ & $17: 13$ & & \\
\hline 37 & $\uparrow 18: 48$ & $5: 28$ & $16: 20$ & $\downarrow 6: 33$ & 112 & $7: 49$ & $15: 23$ & & \\
\hline 38 & $\uparrow 16: 50$ & 7:06 & $22: 23$ & $\downarrow 7: 43$ & 113 & $12: 59$ & $18: 57$ & & \\
\hline 39 & $\uparrow 20: 24$ & $4: 44$ & & & 114 & $13: 23$ & 19:01 & & \\
\hline 40 & $17: 43$ & $\downarrow 9: 06$ & & & 115 & $\uparrow 19: 31$ & $8: 56$ & 16:01 & $\downarrow 5: 58$ \\
\hline 41 & $\uparrow 17: 38$ & 9:01 & $20: 26$ & $\downarrow 9: 58$ & 116 & $\uparrow 18: 15$ & $7: 46$ & $16: 02$ & $\downarrow 5: 12$ \\
\hline 42 & $\uparrow 17: 21$ & 7:01 & $17: 36$ & $\downarrow 11: 05$ & 117 & $7: 28$ & $15: 45$ & & \\
\hline
\end{tabular}


Table A1. Cont.

\begin{tabular}{|c|c|c|c|c|c|c|c|c|c|}
\hline \multirow[b]{2}{*}{ No. } & \multicolumn{2}{|c|}{ First Charge } & \multicolumn{2}{|c|}{ Second Charge } & \multirow[b]{2}{*}{ No. } & \multicolumn{2}{|c|}{ First Charge } & \multicolumn{2}{|c|}{ Second Charge } \\
\hline & $\begin{array}{l}\text { On-Grid } \\
\text { Time }\end{array}$ & $\begin{array}{l}\text { Off-Grid } \\
\text { Time }\end{array}$ & $\begin{array}{l}\text { On-Grid } \\
\text { Time }\end{array}$ & $\begin{array}{l}\text { Off-Grid } \\
\text { Time }\end{array}$ & & $\begin{array}{l}\text { On-Grid } \\
\text { Time }\end{array}$ & $\begin{array}{l}\text { Off-Grid } \\
\text { Time }\end{array}$ & $\begin{array}{l}\text { On-Grid } \\
\text { Time }\end{array}$ & $\begin{array}{l}\text { Off-Grid } \\
\text { Time }\end{array}$ \\
\hline 43 & १17:27 & $9: 57$ & $17: 45$ & $\downarrow 4: 45$ & 118 & $\uparrow 17: 16$ & $7: 59$ & $18: 26$ & $\downarrow 10: 10$ \\
\hline 44 & $7: 52$ & $16: 33$ & & & 119 & $\uparrow 22: 34$ & $8: 32$ & $16: 48$ & $\downarrow 7: 09$ \\
\hline 45 & $\uparrow 16: 10$ & 7:07 & $18: 35$ & $\downarrow 6: 14$ & 120 & 9:31 & $15: 45$ & & \\
\hline 46 & $\uparrow 18: 23$ & 10:02 & $17: 59$ & $\downarrow 9: 29$ & 121 & $16: 41$ & $\downarrow 6: 49$ & & \\
\hline 47 & $\uparrow 16: 51$ & $9: 16$ & $16: 39$ & $\downarrow 7: 41$ & 122 & $16: 26$ & $\downarrow 7: 12$ & & \\
\hline 48 & $\uparrow 17: 09$ & $9: 21$ & & & 123 & $12: 48$ & $16: 48$ & & \\
\hline 49 & $\uparrow 18: 32$ & $8: 23$ & $18: 20$ & $\downarrow 8: 26$ & 124 & $\uparrow 14: 02$ & $9: 30$ & $16: 44$ & $\downarrow 8: 45$ \\
\hline 50 & $\uparrow 21: 36$ & $6: 10$ & $17: 06$ & $\downarrow 10: 16$ & 125 & $13: 32$ & $18: 26$ & & \\
\hline 51 & $9: 55$ & $17: 28$ & & & 126 & $\uparrow 17: 25$ & $8: 40$ & $20: 45$ & $\downarrow 6: 57$ \\
\hline 52 & $\uparrow 17: 41$ & $8: 41$ & $18: 35$ & $\downarrow 7: 08$ & 127 & $\uparrow 18: 53$ & 9:00 & $19: 54$ & $\downarrow 6: 59$ \\
\hline 53 & $\uparrow 17: 19$ & $8: 21$ & 22:21 & $\downarrow 5: 53$ & 128 & $\uparrow 17: 17$ & $8: 18$ & 20:15 & $\downarrow 7: 49$ \\
\hline 54 & $\uparrow 18: 12$ & $4: 51$ & $18: 47$ & $\downarrow 9: 37$ & 129 & $\uparrow 18: 50$ & $6: 01$ & $18: 34$ & $\downarrow 9: 47$ \\
\hline 55 & $10: 29$ & $15: 57$ & & & 130 & 8:05 & $15: 52$ & & \\
\hline 56 & $\uparrow 18: 49$ & $6: 56$ & 19:30 & $\downarrow 8: 17$ & 131 & $6: 37$ & $16: 29$ & & \\
\hline 57 & $\uparrow 20: 8$ & 9:08 & $20: 44$ & $\downarrow 8: 21$ & 132 & $8: 10$ & $18: 31$ & & \\
\hline 58 & 9:48 & $16: 36$ & & & 133 & $\uparrow 17: 46$ & $6: 39$ & 18:03 & $\downarrow 6: 13$ \\
\hline 59 & $17: 48$ & $\downarrow 9: 48$ & & & 134 & $\uparrow 18: 27$ & $6: 45$ & $18: 17$ & $\downarrow 5: 44$ \\
\hline 60 & $\uparrow 21: 24$ & 8:07 & & & 135 & $\uparrow 18: 42$ & $7: 27$ & $16: 21$ & $\downarrow 7: 15$ \\
\hline 61 & $\uparrow 19: 50$ & $8: 12$ & & & 136 & $\uparrow 19: 22$ & $8: 38$ & $15: 49$ & $\downarrow 9: 53$ \\
\hline 62 & $8: 40$ & $18: 28$ & & & 137 & $\uparrow 17: 36$ & $10: 14$ & $21: 50$ & $\downarrow 9: 26$ \\
\hline 63 & $9: 34$ & $15: 37$ & & & 138 & $\uparrow 18: 39$ & $7: 27$ & $17: 59$ & $\downarrow 7: 30$ \\
\hline 64 & $12: 45$ & $17: 10$ & & & 139 & $\uparrow 19: 58$ & $4: 13$ & $20: 07$ & $\downarrow 7: 46$ \\
\hline 65 & $\uparrow 20: 16$ & $6: 01$ & $17: 50$ & $\downarrow 9: 18$ & 140 & $\uparrow 17: 40$ & 9:33 & 18:06 & $\downarrow 10: 14$ \\
\hline 66 & $\uparrow 19: 32$ & $5: 40$ & $18: 15$ & $\downarrow 9: 31$ & 141 & $\uparrow 22: 19$ & $6: 33$ & $19: 44$ & $\downarrow 8: 27$ \\
\hline 67 & $\uparrow 20: 06$ & $9: 33$ & & & 142 & $\uparrow 18: 40$ & $5: 36$ & $10: 29$ & 19:09 \\
\hline 68 & $\uparrow 19: 15$ & 7:00 & $15: 10$ & $\downarrow 7: 17$ & 143 & $\uparrow 18: 55$ & $6: 26$ & 18:06 & $\downarrow 5: 09$ \\
\hline 69 & 10:32 & $17: 19$ & & & 144 & $10: 29$ & $16: 26$ & & \\
\hline 70 & $12: 48$ & $16: 31$ & & & 145 & $\uparrow 17: 54$ & 10:14 & & \\
\hline 71 & $\uparrow 17: 37$ & 9:49 & $17: 16$ & $\downarrow 9: 28$ & 146 & $8: 57$ & $17: 56$ & & \\
\hline 72 & $\uparrow 16: 02$ & $6: 18$ & 19:50 & $\downarrow 8: 21$ & 147 & 9:50 & $15: 10$ & & \\
\hline 73 & $\uparrow 18: 01$ & $6: 16$ & $16: 52$ & $\downarrow 7: 39$ & 148 & $12: 43$ & 19:49 & & \\
\hline 74 & $8: 34$ & $15: 30$ & & & 149 & $\uparrow 22: 47$ & $4: 54$ & $16: 21$ & $\downarrow 5: 29$ \\
\hline 75 & $\uparrow 20: 24$ & $6: 18$ & $20: 00$ & $\downarrow 7: 22$ & 150 & $\uparrow 16: 38$ & $8: 43$ & & \\
\hline
\end{tabular}




\section{References}

1. Mozina, C. Impact of green power distributed generation. J. IEEE Ind. Appl. Mag. 2010, 16, 55-62. [CrossRef]

2. Zhang, Q.; Tezuka, T.; Esteban, M. A study of renewable power for a zero-carbon electricity system in Japan using a proposed integrated analysis model. In Proceedings of the 2nd International Conference on Computer and Automation Engineering, Singapore, 26-28 February 2010; pp. 166-170.

3. Vilayanur, V.V.; Michael, K.M. Second use of transportation batteries: Maximizing the value of batteries for transportation and grid services. J. IEEE Trans. Veh. Technol. 2011, 60, 2963-2970.

4. Sun, Y.; Huang, X.L.; Chen, Z. Time-scale collaborative optimal dispatch methods for electric vehicles and wind power. J. Appl. Mech. Mater. 2014, 472, 958-964. [CrossRef]

5. Cheng, L.; Ma, R.; Lu, H.W. Research on collaborative operation system of wind power and electric vehicle. In Proceedings of the 2nd International Conference on Applied Mechanics, Materials and Manufacturing, Changsha, China, 17-18 November 2012; pp. 168-170.

6. Zhao, J.H.; Wen, F.; Dong, Z.Y. Optimal dispatch of electric vehicles and wind power using enhanced particle swarm optimization. J. IEEE Trans. Ind. Inform. 2012, 8, 889-899. [CrossRef]

7. Vasirani, M.; Kota, R.; Cavalcante, R. An agent-based approach to virtual power plants of wind power generators and electric vehicles. J. IEEE Trans. Smart Grid 2013, 4, 1314-1322. [CrossRef]

8. Li, L.S.; Jin, W.C.; Shen, M.Y. Coordinated dispatch of integrated energy systems considering the differences of multiple functional areas. J. Appl. Sci. 2019, 9, 2103. [CrossRef]

9. Zhang, N.; Hu, Z.G.; Han, X. A fuzzy chance-constrained program for unit commitment problem considering demand response, electric vehicle and wind power. Int. J. Electr. Power Energy Syst. 2015, 65, 201-209. [CrossRef]

10. Omran, N.G.; Filizadeh, S. Location-based forecasting of vehicular charging load on the distribution system. J. IEEE Trans. Smart Grid 2014, 5, 632-641. [CrossRef]

11. Deilami, S.; Masoum, A.S.; Moses, P.S. Real-time coordination of plug-in electric vehicle charging in smart grids to minimize power losses and improve voltage profile. J. IEEE Trans. Smart Grid 2011, 2, $456-467$. [CrossRef]

12. Luo, X.; Chan, K.W. Real-time scheduling of electric vehicles charging in low-voltage residential distribution systems to minimise power losses and improve voltage profile. J. IET Gener. Transm. Distrib. 2014, 8, 516-529. [CrossRef]

13. Zhang, Y.B.; Liu, Q.H.; Hong, C.W.; Tang, G.Y. Charging and discharging dispatch strategy of regional V2G based on fuzzy control. J. Electr. Power Autom. Equip. 2019, 39, 147-152.

14. Su, H.F.; Liang, Z.R. Orderly charging control based on peak-valley electricity tariffs for household electric vehicles of residential quarter. J. Electr. Power Autom. Equip. 2015, 35, 17-22.

15. Wang, Y.; Wang, F.H.; Hou, X.Z. Random access control strategy of charging for household electric vehicle in residential area. J. Autom. Electr. Power Syst. 2018, 42, 53-58.

16. Hu, J.J.; Zhou, H.Y.R.; Li, Y. Real-time dispatching strategy for aggregated electric vehicles to smooth power fluctuation of photovoltaics. J. Power Syst. Technol. 2019, 43, 2552-2560.

17. Pahasa, J.; Ngamroo, I. PHEVs bidirectional charging/discharging and soc control for microgrid frequency stabilization using multiple MPC. J. IEEE Trans. Smart Grid 2015, 6, 526-533. [CrossRef]

18. Geng, B.; Mills, J.K.; Sun, D. Two-stage charging strategy for plug-in electric vehicles at the residential transformer level. J. IEEE Trans. Smart Grid 2013, 4, 1442-1452. [CrossRef]

19. Sun, G.Z.; Sun, Q.; Yang, W.T. Research and Design of DC Charging System for Electric Vehicle. In Proceedings of the International Conference on Mechanical, Electronic and Information Technology, Shanghai, China, 15-16 April 2018; pp. 79-83.

20. Wang, X.; He, Z.Y.; Yang, J.W. Electric vehicle fast-charging station unified modeling and stability analysis in the dq frame. J. Energies 2018, 11, 1195. [CrossRef]

21. Xu, Z.W.; Callaway, D.S.; Hu, Z.C.; Song, Y.H. Hierarchical coordination of heterogeneous flexible loads. J. IEEE Trans. Power Syst. 2016, 31, 4206-4216. [CrossRef]

22. Paatero, V.J.; Lund, P.D. Effect of energy storage on variations in wind power. J. Wind Energy 2005, 8, 421-441. [CrossRef]

23. Díaz-González, F.; Sumper, A.; Gomis-Bellmunt, O.; Bianchi, F.D. Energy management of flywheel-based energy storage device for wind power smoothing. J. Appl. Energy 2013, 110, 207-219. [CrossRef] 
24. Lofberg, J. YALMIP: A toolbox for modeling and optimization in MATLAB. In Proceedings of the IEEE International Conference on Robotics and Automation, New Orleans, LA, USA, 2-4 September 2004; pp. 287-292.

25. Tao, S.; Liao, K.Y.; Xiao, X.N. Charging demand for electric vehicle based on stochastic analysis of trip chain. J. IET Gener. Transm. Distrib. 2016, 10, 2689-2698.

26. Gong, W.; Liu, J.Y.; He, X.Q.; Liu, Y. Load restoration considering load fluctuation rate and load complementary coefficient. J. Power Syst. Technol. 2014, 38, 2490-2496.

(C) 2020 by the authors. Licensee MDPI, Basel, Switzerland. This article is an open access article distributed under the terms and conditions of the Creative Commons Attribution (CC BY) license (http://creativecommons.org/licenses/by/4.0/). 